\title{
Estimation of the specific combining ability (SCA) of eight bitter gourd (Momordica charantia L.) inbred lines in the sixth generation
}

\author{
Trinh T. T. To ${ }^{1 *}$, Hiet D. Hoang ${ }^{1}$, Man T. T. Le ${ }^{1},{\text { Lam V. } \operatorname{Tran}^{1}, \text { Phuong T. B. Nguyen }}^{1}$ \\ Tuan Q. Huynh ${ }^{1}$, Bich T. Thai, \& Dan T. Vo ${ }^{2}$ \\ ${ }^{1}$ Research and Development Center for Hi-tech Agriculture, Ho Chi Minh City, Vietnam \\ ${ }^{2}$ Faculty of Agronomy, Nong Lam University, Ho Chi Minh City, Vietnam
}

ARTICLE INFO
Research Paper
Received: February 01, 2021
Revised: April 02, 2021
Accepted: April 20, 2021
Keywords
Bitter gourd
Characteristics
Momordica charantia L.
Specific combining ability (SCA)
Yield

*Corresponding author

To Thi Thuy Trinh

Email: trinhtocnc@gmail.com

\begin{abstract}
Estimating the Specific Combining Ability (SCA) of bitter gourd (Momordica charantia L.) inbred lines for breeding F1 hybrid cultivars was performed at the Research and Development Center for Hi-tech Agriculture in Ho Chi Minh City from September to December 2019. The objective of the study was to select the outstanding hybrid combinations from diallen method to produce F1 hybrid cultivars. The results from estimating the SCAs based on the productivity characteristic of 28 hybrid combinations produced from eight bitter gourd I6 inbred lines (Q16, Q65, Q80, Q86, Q75, Q06, Q81 và Q87) following diallen method showed that four promised hybrid combinations (Q65/Q80, Q86/Q75, Q06/Q81 and Q81/Q87) performed over $10 \%$ higher SCA values and heterosis than the control, and they were less susceptible to infectious diseases. Fruit characteristics and yield of four hybrid combinations included fruit length of 16.0 to $17.6 \mathrm{~cm}$; fruit width of 6.2 to $6.8 \mathrm{~cm} ; 132.2$ to $151.7 \mathrm{~g} /$ fruit and average yield of 4.1 to 4.3 tons $/ 1000 \mathrm{~m}^{2}$.
\end{abstract}

Cited as: To, T. T. T, Hoang, H. D., Le, M. T. T., Tran, L. V., Nguyen, P. T. B., Huynh, T. Q., Thai, B. T., \& Vo, D. T. (2021). Estimation of the specific combining ability (SCA) of eight bitter gourd (Momordica charantia L.) inbred lines in the sixth generation. The Journal of Agriculture and Development 20(2), 17-26. 


\title{
Đánh giá khả năng kết hợp riêng của 8 dòng khổ qua (Momordica charantia L. ) thế hệ $\mathbf{I}_{6}$
}

\author{
Tô Thị Thùy Trinh ${ }^{1 *}$, Hoàng Đắc Hiệt ${ }^{1}$, Lê Thị Thu Mận ${ }^{1}$, Trần Văn Lâm ${ }^{1}$, \\ Nguyễn Thị Bích Phượng ${ }^{1}$, Huỳnh Quang Tuấn ${ }^{1}$, Thái Thị Bích ${ }^{1}$ \& Võ Thái Dân ${ }^{2}$ \\ ${ }^{1}$ Trung Tâm Nghiên Cứu và Phát Triển Nông Nghiệp Công Nghệ Cao, TP. Hồ Chí Minh \\ ${ }^{2}$ Khoa Nông Học, Trường Đại Học Nông Lâm TP.HCM, TP. Hồ Chí Minh
}

\section{THÔNG TIN BÀI BÁO}

Bài báo khoa học

Ngày nhận: 01/02/2021

Ngày chỉnh sưa: 02/04/2021

Ngày chấp nhận: 20/04/2021

Từ khóa

Cây khổ qua

Hình thái

Khả năng kết hợp riêng (SCA) Momordica charantia L.

Năng suất

*Tác giả liên hệ

Tô Thị Thùy Trinh

Email: trinhtocnc@gmail.com

\section{TÓM TẮT}

Đánh giá khả năng kết hợp riêng của 8 dòng khổ qua (Momordica charantia L.) thế hệ $\mathrm{I}_{6}$ được thực hiện tại Trung tâm Nghiên cứu và Phát triển Nông nghiệp Công nghệ cao trong thời gian từ tháng 09/2019 đến tháng 12/2019. Mục tiêu là chọn tổ hợp lai ưu tú bằng phương pháp lai diallen phục vụ lai tạo giống F1. Kết quả đánh giá khả năng phối hợp riêng dựa trên tính trạng năng suất của 28 tổ hợp lai bằng phương pháp lai diallen của 8 dòng khổ qua thế hệ I6 (Q16, Q65, Q80, Q86, Q75, Q06, Q81 và Q87), chọn được 04 tổ hợp lai triển vọng có ưu thế lai cao vượt giống đối chứng từ $10 \%$ trở lên phục vụ công tác sản xuất gồm Q65/Q80, Q86/Q75, Q06/Q81 và Q81/Q87) và ít bị nhiễm bệnh hại. Đặc điểm quả và năng suất của bốn tổ hợp lai được chọn: chiều dài quả từ 16,0 - 17,6 cm; đường kính quả từ $6,2-6,8 \mathrm{~cm}$; khối lượng quả trung bình dao động từ 132,2 - 151,7 g/quả và năng suất trung bình đạt 4,1 đến 4,3 tấn $/ 1000 \mathrm{~m}^{2}$.

\section{1. Đặt Vấn Đề}

Cây khổ qua (Momordica charantia L.), thuộc họ bầu bí (Cucurbitaceae), là cây trồng phổ biến tại Việt Nam và một số quốc gia khác như Ân Độ, Philippines, Trung Quốc, các nước ở châu Phi, Tây Á và Mỹ La Tinh, góp phần mang lại thu nhập cho nông dân. Sản phẩm từ cây khổ qua được sử dụng làm thực phẩm trong các bữa ăn hàng ngày và được chế biến thành các loại thực phẩm chức năng như trà thảo dược có lợi cho sức khỏe.

Tại Việt Nam, các giống khổ qua đang được nông dân sản xuất chủ yếu là giống lai F1 được nhập nội. Giống F1 cho năng suất cao, có thể trồng quanh năm nhưng giá thành hạt giống cao và không chủ động được nguồn hạt. Nghiên cứu lai tạo hạt giống F1 trong nước góp phần chủ động nguồn hạt giống trong nước và hạ giá thành của hạt giống. Tùy theo thị hiếu của người tiêu dùng mà mục đích lai tạo giống phù hợp với từng thị trường. Đối với vùng Đông nam bộ chọn giống lai $\mathrm{F} 1$ có chiều dài quả trung bình, dạng quả thuôn, màu xanh nhạt, thịt dày có vị đắng ít và sức sinh trưởng mạnh, kháng bệnh phấn trắng, trồng quanh năm (Phan, 2017).

Nhận thấy tiềm năng của hạt giống lai F1 trong việc sản xuất các loại rau quả nói chung và giống cây khổ qua nói riêng, từ năm 2016, Trung tâm Nghiên cứu và phát triển Nông nghiệp Công nghệ cao đã thực hiện thu thập những mẫu giống khổ qua trồng tại khu vực Đông Nam bộ và tiến hành chọn lọc được 8 dòng khổ qua thế hệ I6 phù hợp với mục tiêu của sản xuất hạt giống lai F1 (To \& ctv., 2019). Mục tiêu là tạo ra các tổ hợp lai ưu tú, có khả năng sinh trưởng và năng suất cao để sản xuất ra hạt giống lai F1. Do đó, nghiên cứu "Đánh giá khả năng kết hợp riêng của 8 dòng khổ 
qua (Momordica charantia L.) thế hệ $\mathrm{I}_{6}$ " đã được thực hiện.

\section{Vật Liệu và Phương Pháp Nghiên Cứu}

\subsection{Vật liệu}

Tám dòng khổ qua thuần được ký hiệu (Bảng 1): Q16, Q65, Q80, Q86, Q75, Q06, Q81 và Q87. 28 tổ hợp lai được tạo thành bằng phương pháp lai diallen theo chiều thuận (phương pháp IV) tạo thành số tổ hợp lai theo công thức: $\mathrm{p}(\mathrm{p}-1) / 2=$ $8(8-1) / 2=28$ tổ hợp lai (Griffing, 1956). Giống đối chứng là giống lai $\mathrm{F} 1$ Vino 606 của công ty Việt Nông.

Thành phần giá thể trồng cây khổ qua gồm mụn dừa và phân trùn quế. Mụn dừa được xử lý sạch chất chát bằng cách ngâm xả nước trong 7 10 ngày, các chỉ số của mụn dừa đạt để đưa vào trồng cây như sau: độ pH: 5,5; Lignin: 62 - 75\%; Tanin: 8,0 - 8,8\%; EC: 0,07 dS/m. Phân trùn quế gồm chất hữu cơ (\%): C: 7,64; mùn: 13,17; chất tổng số (\%): N: 1,93: P: 0,71; K: 0,70 (AHRD, 2015).

Các loại hóa chất pha dung dịch thủy canh theo quy trình trồng khổ qua của Trung tâm Nghiên cứu và Phát triển Nông nghiệp Công nghệ cao: Monopotassium phosphate $\left[\mathrm{KH}_{2} \mathrm{PO}_{4}\right]$ $(23 \% \mathrm{P}$ và $28 \% \mathrm{~K}$; Jordan), Calcium nitrate $\left[\mathrm{Ca}\left(\mathrm{NO}_{3}\right) 2 \cdot 4 \mathrm{H}_{2} \mathrm{O}\right](16 \% \mathrm{~N}$ và $20 \% \mathrm{Ca}$; Jordan $)$, Potassium sulfate $\left[\mathrm{K}_{2} \mathrm{SO}_{4}\right](43,3 \% \mathrm{~K}$; Jordan), Magnesium sulfate [MgSO4.7 $\left.\mathrm{H}_{2} \mathrm{O}\right](11 \% \mathrm{Mg}$; Jordan), Solubor $\left[\mathrm{H}_{3} \mathrm{BO}_{3}\right](20,5 \%$ B; Ấn Độ), Zinc sulfate $\left[\mathrm{ZnSO}_{4}\right](36 \% \mathrm{Zn}$; Ân Độ), Copper sulfate $\left[\mathrm{CuSO}_{4} .5 \mathrm{H}_{2} 0\right](25 \% \mathrm{Cu}$; Ân Độ), Sodium molybdate $\left[\mathrm{Na}_{2} \mathrm{MoH}_{2} \mathrm{O}\right](39,6 \% \mathrm{Mo}$; Đức) và Chelated sắt (11\% Fe; Ấn Độ). Hóa chất sử dụng trong thí nghiệm có độ tinh khiết $89 \%$.

\section{2. Điều kiện thí nghiệm}

Thời gian thí nghiệm: 05/09 đến 15/12/2019.

Thí nghiệm được thực hiện trong điều kiện nhà màng kiểu máy thông gió cố định, hệ thống tưới nhỏ giọt theo thiết kế của Trung tâm Nghiên cứu và Phát triển Nông nghiệp Công nghệ cao năm 2015. Bên trong nhà màng được thiết kế một lớp lưới cắt nắng $50 \%$. Trung bình nhiệt độ trong nhà màng trong thời gian thí nghiệm ghi nhận được là $30,1-34,8^{\circ} \mathrm{C}$. Độ ẩm không khí trong nhà màng trong thời gian thí nghiệm là $64,3-75,2 \%$, thích

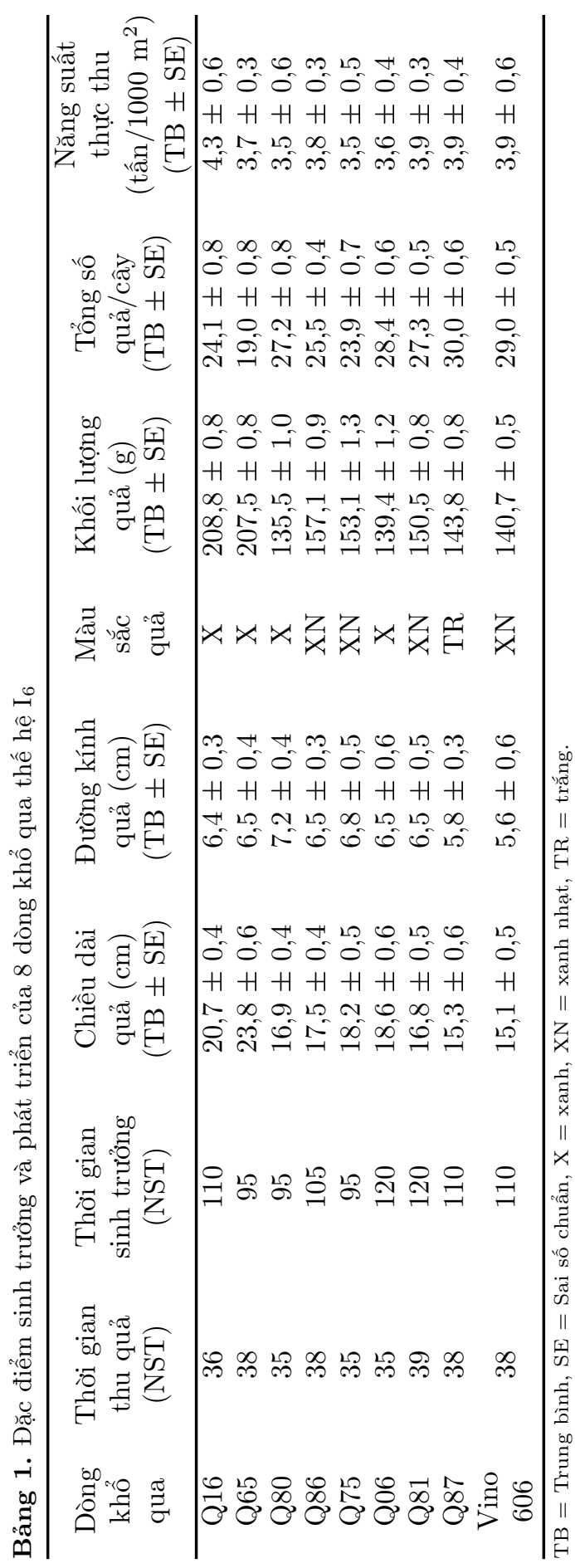

Tạp chí Nông nghiệp và Phát triển 20(2) 
hợp cho khổ qua.

\subsection{Phương pháp nghiên cứu}

Thí nghiệm đơn yếu tố được bố trí kiểu hoàn toàn ngẫu nhiên (CRD), 37 nghiệm thức (gồm 28 tổ hợp lai, 1 giống đối chứng Vino 606 và 8 dòng bố mẹ) và 3 lần lặp lại. Mỗi ô thí nghiệm trồng 50 cây thành 1 hàng. Khoảng cách trồng 1,4 x 0,5 $\mathrm{m}$. Tiến hành gieo ươm cây con trong khay xốp và trồng khi cây con có 2 lá thật, đạt 8 ngày sau gieo. Giá thể để trồng và ườm cây gồm $70 \%$ mụn dừa $+20 \%$ phân trùn quế và $10 \%$ tro trấu. Cây được trồng trong bầu với thể tích $5 \mathrm{dm}^{3}$ giá thể.

Các chỉ tiêu theo dõi: thời gian thu quả (ngày), thời gian sinh trưởng (ngày), số cành cấp 1 , chiều dài quả $(\mathrm{cm})$, đường kính quả $(\mathrm{cm})$, màu sắc quả, hình dạng quả, gai quả, độ đắng của quả: (đánh giá theo phương pháp cảm quan theo thang điểm từ 1 - 5) ((1) rất đắng; $(2-4)$ đắng trung bình; (5) không đắng)), tổng số quả/cây (quả), khối lượng trung bình quả $(\mathrm{g})$, năng suất loại $1\left(\mathrm{~kg} / 1000 \mathrm{~m}^{2}\right)$ và năng suất thực thu $\left(\mathrm{kg} / 1000 \mathrm{~m}^{2}\right)$, theo dõi tỷ lệ nhiễm bệnh sương mai (Pseudoperonospora sp.) (\%), bệnh phấn trắng (Erysiphe sp.) (\%) và vi rút (\%) (theo tiêu chuẩn ngành 10 TCN 224:2003).

Nội dung được tiến hành trong điều kiện nhà màng, chăm sóc theo quy trình kỹ thuật trồng khổ qua trên giá thể trong nhà màng. Giai đoạn cây từ trồng đến ra hoa 100 ppm N, 50 ppm P, 80 ppm K, ppm $50 \mathrm{Mg}, 90$ ppm Ca. Giai đoạn cây từ ra hoa đến hết vụ 160 ppm N, 60 ppm P, 250 ppm K, ppm 50 Mg, 130 ppm Ca. Nồng độ phân vi lượng bổ sung $0,3-0,5$ ppm B, 0,3 ppm Mn, 2 - 3 ppm Fe, 0,05 ppm Mo, 0,1 - 0,5 ppm Cu, 0,3 ppm Zn (AHRD, 2016).

\subsection{Phương pháp đánh giá khả năng phối hợp}

Xác định mức độ ưu thế lai gồm ưu thế lai trung bình, ưu thế lai thực, ưu thế lai chuẩn. Đánh giá khả năng phối hợp riêng yếu tố năng suất thực (Phan, 2006).

\subsection{Phương pháp xử lý số liệu}

Số liệu thí nghiệm được phân tích ANOVA, phân hạng trên phần mềm SAS 9.1. Khả năng phối hợp riêng được tính toán trên phần mềm Microsoft Excel và xây dựng cây phát sinh loài bằng phần mềm NTSYSpc 2.1.

\section{Kết Quả và Thảo Luận}

\section{1. Đặc điểm sinh trưởng của 28 tổ hợp lai được lai từ 08 dòng khổ qua thế hệ $\mathbf{I}_{6}$}

Kết quả của lai tạo 8 dòng tự phối giống khổ qua bằng phương pháp lai diallen thu được 28 tổ hợp lai. Ghi nhận được tất cả các tổ hợp lai đều có khả năng hình thành hạt, tỷ lệ đậu quả và cho ra hạt giống trên $75 \%$. Tỷ lệ hạt nảy mầm đạt từ $71 \%$ đến $92 \%$, thời gian cho thu trung bình của các tổ hợp lai là 35 ngày, thời gian quả sớm nhất là 33,4 ngày và muộn nhất là 40,3 ngày. Các tổ hợp lai có khả năng phân cành mạnh, số cành cấp 1 dao động từ 19,8 đến 30,4 cành/cây. Thời gian sinh trưởng của các tổ hợp lai từ 94,1 đến 120,6 ngày sau trồng.

\section{2. Đặc điểm quả của 28 tổ hợp lai được lai từ 08 dòng khổ qua thế hệ $\mathbf{I}_{6}$}

Theo kết quả Bảng 2, các tổ hợp lai có chiều dài quả trung bình từ $15,5-20,9 \mathrm{~cm}$. Đường kính quả của các con lai thử dao động từ $5,5-7,3 \mathrm{~cm}$. Về đặc điểm kiểu hình quả qua theo dõi các tổ hợp lai, quả có dạng thuôn, màu sắc quả từ xanh nhạt đến xanh (Hình 1). Đặc điểm gai quả của các giống khổ qua được mô tả theo phương pháp cảm quan, quả các tổ hợp lai có dạng gai nở, sóng gai liền và ít $u$ vấu. Giống đối chứng Vino 606 có chiều dài quả trung bình $15,1 \mathrm{~cm}$ và đường kính quả 5,6 cm, quả dạng thuôn, màu xanh nhạt, gai nở, sóng gai liền và ít $u$ vấu. Tính trạng chiều dài quả và màu sắc quả có dạng trung gian của bố và mẹ, màu xanh nhạt là dạng trung gian giữa màu trắng và màu xanh của giống bố mẹ.

Kết quả này cũng tương đồng với kết quả của Tran (2017), màu sắc quả của các tổ hợp lai có quả màu xanh nhạt (màu trung gian) là con lai các dòng có quả màu xanh với dòng thử có quả màu trắng. Đánh giá cảm quan cho thấy các tổ hợp lai có độ đắng trung bình. Theo khảo sát thị hiếu người tiêu dùng của công ty Việt Nông năm 2017, người tiêu dùng miền Đông nam bộ ưa chuộng quả có chiều dài từ $14-16 \mathrm{~cm}$, gai nở, ít $\mathrm{u}$ vấu, thịt dày và có độ đắng trung bình.

3.3. Các yếu tố cấu thành năng suất và năng suất của 28 tổ hợp lai được lai từ 08 dòng khổ qua thế hệ $\mathbf{I}_{6}$

Theo kết quả Bảng 3, khối lượng trung bình quả khổ qua nặng nhất ở tổ hợp lai Q16/Q86 
Bảng 2. . Đặc điểm hình thái quả của 28 tổ hợp lai khổ qua được lai từ 08 dòng khổ qua thế hệ $\mathrm{I}_{6}$

\begin{tabular}{|c|c|c|c|}
\hline Cặp lai & Chiều dài quả $(\mathrm{cm})$ & Đường kính quả (cm) & Màu sắc quả \\
\hline Q16XQ65 & $20,9^{\mathrm{a}}$ & $6,7^{\mathrm{c}-\mathrm{f}}$ & Xanh \\
\hline Q16XQ80 & $17,1^{\mathrm{e}-\mathrm{g}}$ & $6,1^{\mathrm{jkl}}$ & Xanh nhạt \\
\hline Q16XQ86 & $18,6^{\mathrm{b}}$ & $6,8^{\mathrm{b}-e}$ & Xanh \\
\hline Q16XQ75 & $19,1^{\mathrm{b}}$ & $6,9^{\mathrm{bcd}}$ & Xanh \\
\hline Q16XQ06 & $15,1^{\mathrm{kmn}}$ & $5,8^{\operatorname{lmn}}$ & Xanh nhạt \\
\hline Q16XQ81 & $16,3^{\text {ghi }}$ & $6,4^{\mathrm{f}-\mathrm{i}}$ & Xanh \\
\hline Q16XQ87 & $17,2^{\mathrm{e}-\mathrm{g}}$ & $7,1^{\mathrm{ab}}$ & Xanh nhạt \\
\hline Q65XQ80 & $17,2^{\mathrm{e}-\mathrm{g}}$ & $6,8^{\mathrm{b}-\mathrm{e}}$ & Xanh nhạt \\
\hline Q65XQ86 & $16,6^{\mathrm{e}-\mathrm{h}}$ & $7,0^{\mathrm{abc}}$ & Xanh \\
\hline Q65XQ75 & $20,1^{\mathrm{a}}$ & $6,8^{\mathrm{b}-\mathrm{e}}$ & Xanh \\
\hline Q65XQ06 & $14,5^{\mathrm{lm}}$ & $6,3^{\text {hij }}$ & Xanh nhạt \\
\hline Q65XQ81 & $18,6^{\mathrm{b}}$ & $6,5^{\mathrm{e}-\mathrm{g}}$ & Xanh \\
\hline Q65XQ87 & $17,3^{\mathrm{c}-\mathrm{f}}$ & $6,7^{\mathrm{d}-\mathrm{f}}$ & Xanh nhạt \\
\hline Q80XQ86 & $14,3^{\mathrm{m}}$ & $6,2^{\mathrm{ij}}$ & Xanh nhạt \\
\hline Q80XQ75 & $17,0^{\mathrm{e}-\mathrm{h}}$ & $5,5^{\mathrm{n}}$ & Xanh nhạt \\
\hline Q80XQ06 & $17,6^{\mathrm{cd}}$ & $6,1^{\mathrm{jk}}$ & Xanh nhạt \\
\hline Q80XQ81 & $16,1^{\mathrm{h}-\mathrm{k}}$ & $5,5^{\mathrm{n}}$ & Xanh nhat \\
\hline Q80XQ87 & $15,5^{\mathrm{jkl}}$ & $6,2^{\mathrm{ij}}$ & Xanh nhạt \\
\hline Q86XQ75 & $17,6^{\text {cde }}$ & $6,3^{\text {hij }}$ & Xanh \\
\hline Q86XQ06 & $18,2^{\mathrm{bc}}$ & $5,8^{\operatorname{lmn}}$ & Xanh \\
\hline Q86XQ81 & $16,3^{\mathrm{f}-\mathrm{j}}$ & $6,5^{\mathrm{f}-\mathrm{i}}$ & Xanh \\
\hline Q86XQ87 & $15,4^{\mathrm{jkl}}$ & $6,9^{\mathrm{bcd}}$ & Xanh nhạt \\
\hline Q75XQ06 & $16,1^{\mathrm{h}-\mathrm{k}}$ & $7,3^{\mathrm{a}}$ & Xanh \\
\hline Q75XQ81 & $17,2^{\mathrm{c}-\mathrm{g}}$ & $6,7^{\mathrm{d}-\mathrm{g}}$ & Xanh \\
\hline Q75XQ87 & $15,5^{\mathrm{jkl}}$ & $6,9^{\mathrm{bcd}}$ & Xanh nhạt \\
\hline Q06XQ81 & $16,8^{\mathrm{e}-\mathrm{i}}$ & $6,4^{\text {ghi }}$ & Xanh nhạt \\
\hline Q06XQ87 & $15,8^{\mathrm{ijk}}$ & $5,9^{\mathrm{klm}}$ & Xanh nhạt \\
\hline Q81XQ87 & $16,0^{\mathrm{ijk}}$ & $6,5^{\mathrm{f}-\mathrm{i}}$ & Xanh nhạt \\
\hline Vino 606 - DC & $15,1^{\operatorname{lmn}}$ & $5,6^{\mathrm{mn}}$ & Xanh nhạt \\
\hline $\mathrm{CV}(\%)$ & 2,4 & 2,1 & \\
\hline $\mathrm{F}(\mathrm{t})$ & $45,4^{* *}$ & $42,9^{* *}$ & \\
\hline
\end{tabular}

là 220,1 g/quả và Q80/Q87 là 212,6 g/quả khác biệt so với các tổ hợp lai khác và với đối chứng. Các tổ hợp lai còn lại có khối lượng quả nhỏ hơn $200 \mathrm{~g} /$ quả và nhỏ nhất ở tổ hợp lai Q06/Q81 với khối lượng quả 132,2 g/quả. Số quả/cây trung bình quả của các tổ hợp lai trung bình từ 19,0 35,3 quả/cây. Có 6 tổ hợp lai có số quả/cây lớn hơn 30 quả gồm Q65/Q80, Q86/Q75, Q86/Q06, Q06/Q81, Q06/Q87 và Q81/Q87.

Năng suất loại 1 được theo dõi dựa trên phân loại khổ qua loại 1, theo đánh giá phân loại khổ qua (AHRD, 2016). Khổ qua loại 1: là quả có chiều dài đạt từ $10 \mathrm{~cm}$ trở lên, có hình dạng đẹp, gai quả đều, màu quả đồng nhất, trái thẳng không cong. Loại 2: là quả có chiều dài nhỏ hơn $10 \mathrm{~cm}$, quả bị đèo. Theo kết quả Bảng 3 , về năng suất loại 1 của các tổ hợp lai dao động từ $2.180 \mathrm{~kg}-4.157$ $\mathrm{kg} / 1000 \mathrm{~m}^{2}$, sự khác biết rất có ý nghĩa thống kê. Năng suất thực thu của các tổ hợp lai dao động từ $2.339-4.369 \mathrm{~kg} / 1000 \mathrm{~m}^{2}$, sự khác biết rất có ý nghĩa thống kê. Các tổ hợp lai có năng suất cao nhất gồm Q65/Q80, Q86/Q75, Q86/Q06), Q06/Q81, Q06/Q87 và Q81/Q87 khác biệt có ý nghĩa so với nghiệm thức đối chứng và các nghiệm thức khác. Kết quả này cũng tương đồng với kết quả của Tran (2017), các tổ hợp lai giống khổ qua lai tạo phục vụ nhu cầu giống cho vùng Đông nam bộ, các giống có năng suất lớn hơn 30 tấn/ha. 


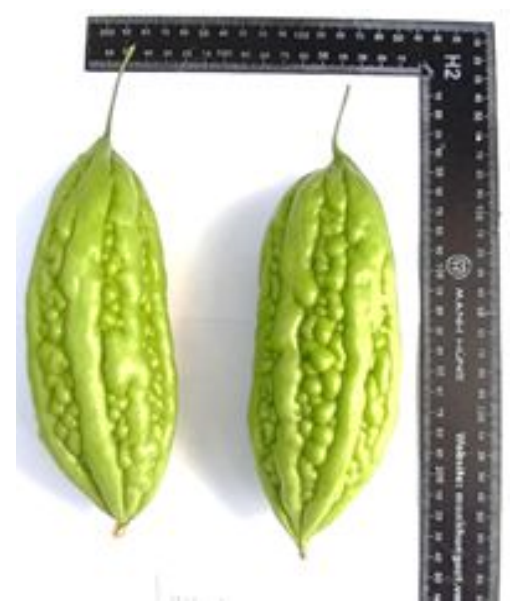

THL 8

Cặp lai Q65XQ80

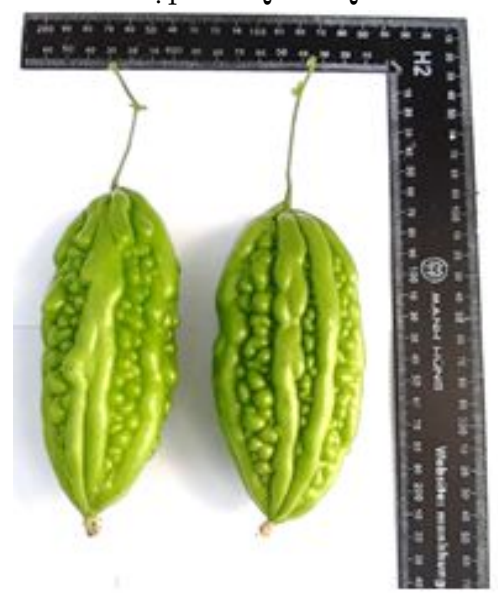

THL 20

Cặp lai Q86XQ06

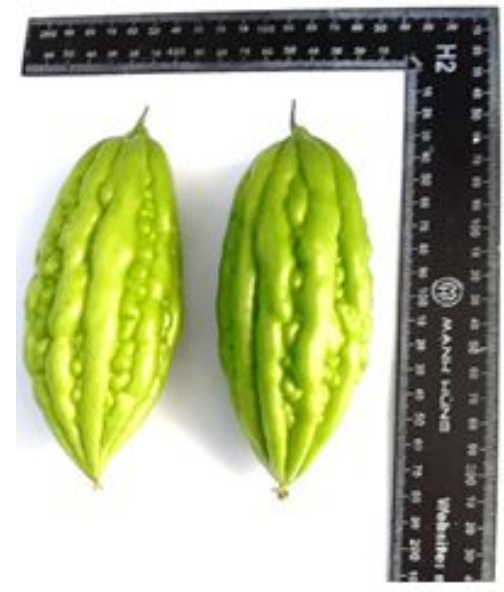

THL 27

Cặp lai Q06XQ87

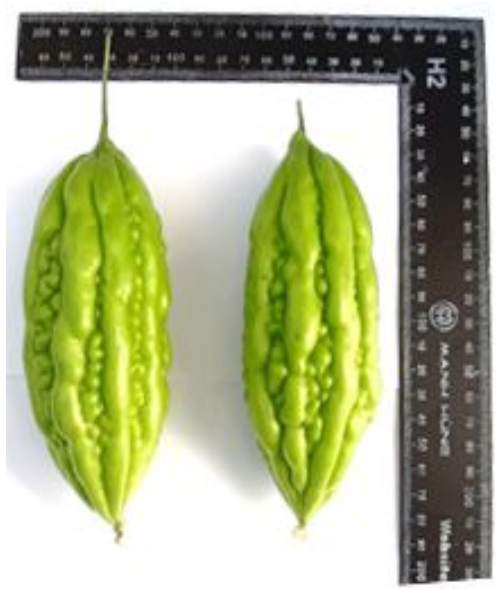

THL 19

Cặp lai Q86XQ75

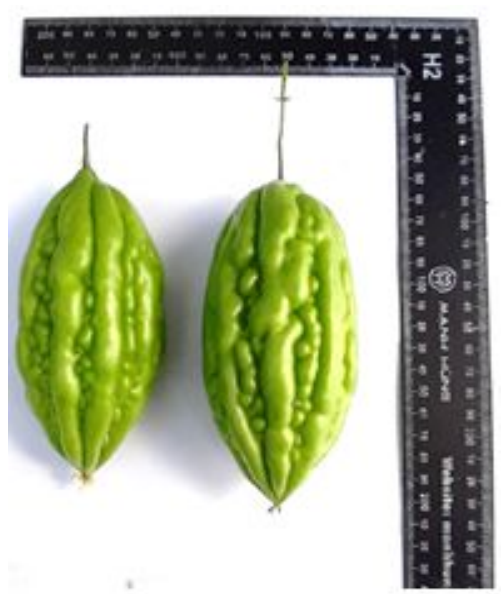

THL 26

Cặp lai Q06XQ81

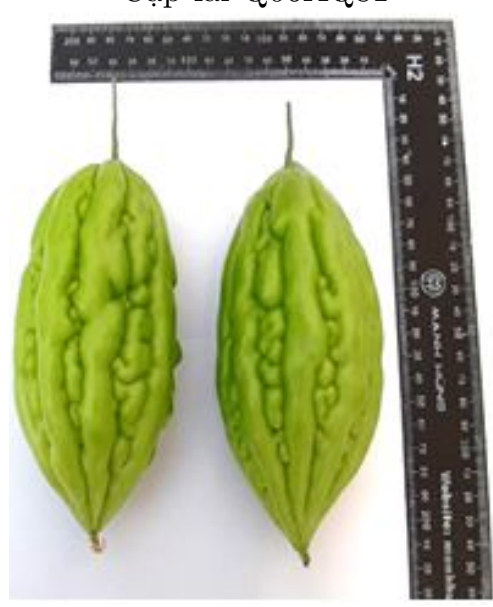

THL 28

Q81XQ87

Hình 1. Đặc điểm quả và hình thái quả của một số tổ hợp lai khổ qua thế hệ $\mathrm{I}_{6}$. 
Bảng 3. Các chỉ tiêu về yếu tố cấu thành năng suất và năng suất của 28 tổ hợp lai được lai từ 08 dòng khổ qua thế hệ $\mathrm{I}_{6}$

\begin{tabular}{|c|c|c|c|c|}
\hline Cặp lai & $\begin{array}{c}\text { Khối lượng } \\
\text { trung bình quả }(\mathrm{g})\end{array}$ & Số quả/cây & $\begin{array}{c}\text { Năng suất loại } 1 \\
\left(\mathrm{~kg} / 1000 \mathrm{~m}^{2}\right)\end{array}$ & $\begin{array}{l}\text { Năng suất thực thu } \\
\left(\mathrm{kg} / 1000 \mathrm{~m}^{2}\right)\end{array}$ \\
\hline Q16/Q65 & $165,7^{\mathrm{d}-\mathrm{g}}$ & $25,8^{\mathrm{e}-\mathrm{g}}$ & $3.538^{\mathrm{bc}}$ & $3.775^{\mathrm{d}-\mathrm{f}}$ \\
\hline Q16/Q80 & $182,6^{\mathrm{bc}}$ & $23,3^{\mathrm{g}-\mathrm{k}}$ & $3.393^{\mathrm{cde}}$ & $3.752^{\mathrm{c}-\mathrm{f}}$ \\
\hline Q16/Q86 & $220,1^{\mathrm{a}}$ & $21,1^{\mathrm{jkl}}$ & $3.546^{\mathrm{bc}}$ & $4.172^{\mathrm{a}-\mathrm{e}}$ \\
\hline Q16/Q75 & $191,1^{\mathrm{bc}}$ & $20,7^{\mathrm{klm}}$ & $3.273^{\text {cde }}$ & $3.559^{\text {fg }}$ \\
\hline Q16/Q06 & $134,9^{\text {no }}$ & $29,3^{\text {cde }}$ & $3.152^{\text {cde }}$ & $3.556^{\mathrm{fg}}$ \\
\hline Q16/Q81 & $159,5^{\mathrm{f}-\mathrm{i}}$ & $26,4^{\mathrm{d}-\mathrm{g}}$ & $3.499^{\mathrm{bcd}}$ & $3.786^{\mathrm{b}-\mathrm{f}}$ \\
\hline Q16/Q87 & $148,1^{\mathrm{i}-\mathrm{m}}$ & $23,3^{\mathrm{g}-\mathrm{k}}$ & $2.825^{\mathrm{ef}}$ & $3.105^{\mathrm{gh}}$ \\
\hline Q65/Q80 & $145,8^{\mathrm{j}-\mathrm{n}}$ & $32,4^{\mathrm{abc}}$ & $4.015^{\mathrm{ab}}$ & $4.241^{\mathrm{ab}}$ \\
\hline Q65/Q86 & $168,7^{\text {def }}$ & $24^{\mathrm{g}-\mathrm{k}}$ & $3.224^{\text {cde }}$ & $3.637^{\text {fg }}$ \\
\hline Q65/Q75 & $164,6^{\mathrm{e}-\mathrm{g}}$ & $24,7^{\mathrm{f}-\mathrm{j}}$ & $3.354^{\text {cde }}$ & $3.602^{\text {fg }}$ \\
\hline Q65/Q06 & $123,4^{\mathrm{f}-\mathrm{i}}$ & $25,7^{\mathrm{e}-\mathrm{g}}$ & $2.612^{\text {fg }}$ & $2.856^{\mathrm{h}}$ \\
\hline Q65/Q81 & $146,1^{\mathrm{j}-\mathrm{n}}$ & $17,7^{\mathrm{m}}$ & $2.180^{\text {fg }}$ & $2.339^{\mathrm{i}}$ \\
\hline Q65/Q87 & $142,1^{\mathrm{k}-\mathrm{o}}$ & $26,9^{\mathrm{d}-\mathrm{g}}$ & $3.186^{\mathrm{g}}$ & $3.447^{\mathrm{fg}}$ \\
\hline $\mathrm{Q} 80 / \mathrm{Q} 86$ & $155,6^{\mathrm{ghi}}$ & $26,4^{\mathrm{d}-\mathrm{g}}$ & $3.364^{\mathrm{cde}}$ & $3.691^{\text {ef }}$ \\
\hline Q80/Q75 & $149^{\mathrm{i}-\mathrm{m}}$ & $27,8^{\text {def }}$ & $3.361^{\mathrm{cde}}$ & $3.728^{\mathrm{def}}$ \\
\hline Q80/Q06 & $155,2^{\mathrm{ghi}}$ & $23,7^{\mathrm{g}-\mathrm{k}}$ & $3.194^{\text {cde }}$ & $3.315^{\text {fgh }}$ \\
\hline Q80/Q81 & $176,4^{\mathrm{cd}}$ & $22,2^{\mathrm{h}-\mathrm{l}}$ & $3.098^{\mathrm{c}-\mathrm{f}}$ & $3.452^{\text {fg }}$ \\
\hline Q80/Q87 & $212,6^{\mathrm{a}}$ & $19,0^{\operatorname{lm}}$ & $3.417^{\mathrm{cd}}$ & $3.636^{\mathrm{fg}}$ \\
\hline Q86/Q75 & $151,7^{\mathrm{i}-\mathrm{l}}$ & $32,1^{\mathrm{abc}}$ & $4.113^{\mathrm{a}}$ & $4.369^{\mathrm{a}}$ \\
\hline Q86/Q06 & $138,4^{\mathrm{mno}}$ & $35,1^{\mathrm{abc}}$ & $4.104^{\mathrm{a}}$ & $4.367^{\mathrm{a}}$ \\
\hline $\mathrm{Q} 86 / \mathrm{Q} 81$ & $163,8^{\mathrm{e}-\mathrm{g}}$ & $24,7^{\mathrm{f}-\mathrm{j}}$ & $3.262^{\text {cde }}$ & $3.625^{\text {fg }}$ \\
\hline Q86/Q87 & $152,0^{\mathrm{i}-\mathrm{l}}$ & $24,1^{\mathrm{g}-\mathrm{k}}$ & $2.998^{\mathrm{def}}$ & $3.238^{\text {fgh }}$ \\
\hline $\mathrm{Q} 75 / \mathrm{Q} 06$ & $149,6^{\mathrm{i}-\mathrm{m}}$ & $25,1^{\mathrm{f}-\mathrm{i}}$ & $3.077^{\mathrm{c}-\mathrm{f}}$ & $3.376^{\text {fg }}$ \\
\hline Q75/Q81 & $172,3^{c d e}$ & $23,5^{\mathrm{g}-\mathrm{k}}$ & $3.281^{\mathrm{cde}}$ & $3.572^{\text {fg }}$ \\
\hline Q75/Q87 & $167,4^{\mathrm{def}}$ & $21,9^{\mathrm{i}-\mathrm{l}}$ & $3.047^{\mathrm{c}-\mathrm{f}}$ & $3.286^{\text {fgh }}$ \\
\hline Q06/Q81 & $132,2^{\text {op }}$ & $35,3^{\mathrm{a}}$ & $3.939^{\mathrm{a}}$ & $4.194^{\mathrm{a}-\mathrm{d}}$ \\
\hline Q06/Q87 & $153,7^{\mathrm{h}-\mathrm{k}}$ & $31,4^{\mathrm{bc}}$ & $4.157^{\mathrm{a}}$ & $4.346^{\mathrm{a}}$ \\
\hline Q81/Q87 & $144,5^{\mathrm{j}-\mathrm{n}}$ & $33,3^{\mathrm{ab}}$ & $3.934^{\mathrm{ba}}$ & $4.256^{\mathrm{abc}}$ \\
\hline Vino $606-\mathrm{DC}$ & $140,7^{\mathrm{l}-\mathrm{o}}$ & $29,7^{\mathrm{cd}}$ & $3.511^{\mathrm{bc}}$ & $3.758^{\mathrm{b}-\mathrm{f}}$ \\
\hline $\mathrm{CV}(\%)$ & 3,0 & 5,6 & 3,538 & 5,7 \\
\hline $\mathrm{F}(\mathrm{t})$ & $62,3^{* *}$ & $29,8^{* *}$ & $14,0^{* *}$ & $15,1^{* *}$ \\
\hline
\end{tabular}

a-p Trong cùng một cột, các trị số có cùng ký tự đi kèm khác biệt không có ý nghĩa về mặt thống kê, ** Khác biệt rất có ý nghĩa (mức $\alpha=0,01)$.

\subsection{Ghi nhận tình hình nhiễm bệnh của 28 tổ hợp lai được lai từ 08 dòng khổ qua thế hệ $\mathbf{I}_{6}$}

Ghi nhận tình hình nhiễm sâu bệnh hại trên 28 tổ hợp lai giống khổ qua cho thấy các tổ hợp lai có sự nhiễm bệnh từ 2,8 đến 38,5\% (Bảng 4). Trong đó 17 tổ họp lai có mức độ nhiễm bệnh nhỏ hơn $10 \%$, có 4 tổ hợp lai có mức độ nhiễm bệnh nhỏ hơn $20 \%$ và 7 tổ hợp lai có mức độ nhiễm bệnh phấn trắng với tỷ lệ bệnh lớn hơn $20 \%$, các tổ hợp lai. Tổ hợp lai có tỷ lệ nhiễm bệnh nhiều nhất là Q75XQ81 38,5\% và tổ hợp lai Q65XQ80 có tỷ lệ bệnh phấn trắng thấp nhất là $2,1 \%$.
Đối với bệnh sương mai, có 6 tổ hợp lai (Q16/Q86, Q16/Q75, Q06/Q87, Q65/Q87, Q86/Q87 và $\mathrm{Q} 75 / \mathrm{Q} 06)$ có tỷ lệ nhiễm bệnh lớn hơn $20 \%$, các tổ hợp lai còn lại chỉ bị nhiễm ít và rất ít. Đối với bệnh vi rút có 3 tổ hợp lai (Q16/Q75, Q65/Q75 và Q86/Q06) bị nhiễm ở ít tỷ lệ bệnh nhỏ hơn $15 \%$, các tổ hợp lai còn lại nhiễm bệnh vi rút rất ít và không ghi nhận thấy bị nhiễm virut. Giống đối chứng Vino 606 có tỷ lệ bệnh sương mai $(12,8 \%)$ và phấn trắng $(7,9 \%)$ và không bị nhiễm vi rút. 
Bảng 4. Tỷ lệ bệnh hại của 28 tổ họp lai được lai từ 08 dòng khổ qua thế hệ $\mathrm{I}_{6}$

\begin{tabular}{cccc}
\hline Cặp lai & $\begin{array}{c}\text { Tỷ lệ bệnh phấn trắng } \\
\text { Erysiphe sp. })(\%)\end{array}$ & $\begin{array}{c}\text { Tỷ lệ bệnh sương mai } \\
\text { (Pseudoperonospora }\end{array}$ sp.) $(\%)$ & $\begin{array}{c}\text { Tỷ lệ bệnh vi rút } \\
(\%)\end{array}$ \\
\hline Q16XQ65 & 8,2 & 12,9 & 0 \\
Q16XQ80 & 28,5 & 9,8 & 0 \\
Q16XQ86 & 5,7 & 35,6 & 0 \\
Q16XQ75 & 6,5 & 25,1 & 10,7 \\
Q16XQ06 & 12,5 & 7,0 & 0 \\
Q16XQ81 & 3,6 & 9,4 & 0 \\
Q16XQ87 & 25,8 & 10,1 & 0 \\
Q65XQ80 & 2,1 & 3,2 & 0 \\
Q65XQ86 & 4,5 & 9,2 & 0 \\
Q65XQ75 & 7,5 & 12,8 & 5 \\
Q65XQ06 & 30,1 & 7,2 & 11,5 \\
Q65XQ81 & 6,1 & 10,8 & 0 \\
Q65XQ87 & 4,5 & 38,3 & 0 \\
Q80XQ86 & 27,5 & 6,3 & 0 \\
Q80XQ75 & 4,5 & 9,6 & 0 \\
Q80XQ06 & 15,8 & 10,2 & 0,2 \\
Q80XQ81 & 3,5 & 8,8 & 0 \\
Q80XQ87 & 5,7 & 8,0 & 0 \\
Q86XQ75 & 3,1 & 1,1 & 0 \\
Q86XQ06 & 5,3 & 10,5 & 5,5 \\
Q86XQ81 & 21,8 & 9,1 & 0 \\
Q86XQ87 & 29,1 & 25,5 & 1,5 \\
Q75XQ06 & 12,5 & 31,6 & 0 \\
Q75XQ81 & 38,5 & 9,7 & 0,7 \\
Q75XQ87 & 2,8 & 8 & 0 \\
Q06XQ81 & 3,9 & 4,7 & 0 \\
Q06XQ87 & 15,3 & 28,4 & 0 \\
Q81XQ87 & 3,5 & 4,1 & 0 \\
Vino 606 - DC & 12,8 & 7,9 & \\
\hline
\end{tabular}

3.5. Khả năng kết hợp riêng của 8 dòng khổ qua thế hệ $\mathbf{I}_{6}$

Khả năng phối hợp riêng là giá trị F1 sau khi đã trừ đi khả năng phối hợp chung của bố và khả năng phối hợp chung của mẹ .

Theo kết quả Bảng 5 về khả năng phối hợp riêng, tính trạng năng suất thực thu cho thấy có 4 tổ hợp lai có giá trị khả năng phối hợp riêng theo hướng dương cao nhất gồm các tồ hợp lai Q65/Q80 (2,3), Q06/Q81 (2,0), Q86/Q75 (2,1) và Q81/Q87 (2,2). Giá trị khả năng phối hợp riêng giữa các tổ hợp lai trong thí nghiệm có sự khác biệt nhau. Theo Verma \& Singh (2014), khả năng phối hợp riêng các dòng khổ qua được đánh giá vào mùa nắng và mùa mưa cho thây bố mẹ có khả năng phối hợp riêng cao có hiệu ứng tích cực làm tăng năng suất đối với tổ hợp lai hình thành.
Kết quả đánh giá ưu thế lai trung bình (HM\%) được so sánh với giá trị trung bình năng suất thực thu của bố và mẹ, các tổ hợp lai có ưu thế lai về năng suất thực thu biến động từ 43,0\% - 10,9\%. Trong đó, có 07/28 tổ hợp lai có ưu thế lai trung bình về năng suất theo hướng dương và các tổ hợp lai có ưu thế lai trung bình cao nhất là Q16/Q86 (0,9\%) Q65/Q80 (9,8\%), Q86/Q75 (10,9\%), Q86/Q06 (5,1\%), Q06/Q81 $(0,3 \%)$ Q06/Q87 $(5,7 \%)$ và Q81/Q87 $(5,2 \%)$ (Bảng 5).

Ưu thế lai thực (HB\%) được so sánh với giá trị năng suất thực thu của bố và mẹ tốt nhất, các tổ hợp lai có ưu thế lai thực về năng suất thực thu biến động từ - 43,4\% - 5,0\%. Trong đó, có $07 / 28$ tổ hợp lai có ưu thế lai thực về năng suất theo hướng dương và các tổ hợp lai có ưu thế lai thực cao nhất là Q16/Q86 $(0,2 \%)$ 


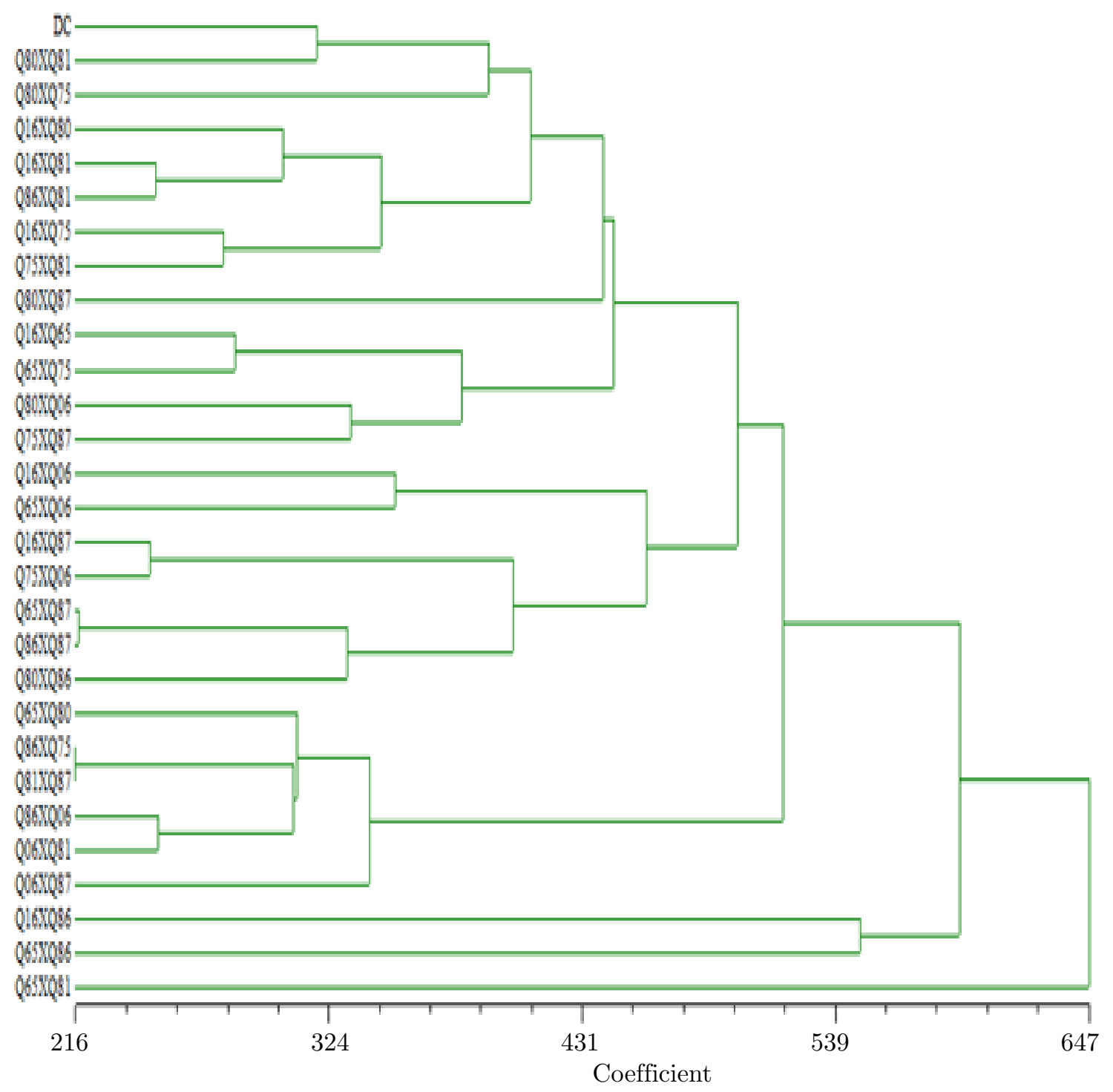

Hình 2. Sơ đồ phân nhóm của cây phát sinh loài của 28 tổ hợp lai được lai từ 08 dòng khổ qua thế hệ $\mathrm{I}_{6}$.

Q65/Q80 (1,9\%), Q86/Q75 (5,0\%), Q86/Q06 $(4,9 \%), \mathrm{Q} 06 / \mathrm{Q} 81 \quad(0,8 \%) \mathrm{Q} 06 / \mathrm{Q} 87 \quad(4,4 \%)$ và Q81/Q87 (4,3\%) (Bảng 5).

Ưu thế lai chuẩn (HS\%) được đánh giá dựa trên việc so sánh năng suất của các tổ hợp lai với năng suất của giống đối chứng. Các tổ hợp lai có ưu thế lai chuẩn biến động từ - 37,8\% - 16,2\%. Trong đó, có $7 / 28$ tổ hợp lai có có ưu thế lai vượt năng suất đối chứng từ $10 \%$ trở lên gồm Q16/Q86 $(11,0 \%)$, Q65/Q80 (12,8\%), Q86/Q75 (16,2\%), Q86/Q06 (16,2\%), Q06/Q81 (11,6\%), Q06/Q87 (15,6\%) và Q81/Q87 (15,5\%) (Bảng 5).

Mối quan hệ di truyền và đa dạng duy truyền của các tổ hợp lai: Các mẫu tổ hợp lai có sự tương đương nhau sẽ xếp chung vào nhóm, dựa vào cây phát sinh loài có thể đánh giá sự tương đồng và đa dạng di truyền giữa các mẫu nghiên cứu. Theo kết quả từ Hình 2 của tổ hợp lai giống khổ qua (được tính toán trên phân mềm NTSYSpc version 2.1), hệ số di truyền có sự tương dồng cao nhất là $0,79 \%$ và thấp nhất là $0,21 \%$. Cây phát sinh loài chia thành 4 nhóm. Nhóm 1 có tổ hợp lai Q65/Q81, nhóm 2 gồm các tổ hợp lai $\mathrm{Q} 16 / \mathrm{Q} 86$ và $\mathrm{Q} 65 / \mathrm{Q} 86$. Nhóm 3 gồm các tổ hợp lai Q65/Q80, Q86/Q75, Q81/Q87, Q86/Q06, Q06/Q81 và 06/87 trong đó tổ hợp lai Q86/Q75, Q81/Q87 có hệ số tương đồng cao nhất $0,79 \%$. Các tổ hợp lai còn lại nằm trong nhóm 4 . 
Bảng 5. Giá trị ưu thế lai của 28 tổ hợp lai

\begin{tabular}{ccccc}
\hline Cạp & KNPHR & $\begin{array}{c}\text { HM } \\
(\%)\end{array}$ & $\begin{array}{c}\text { HB } \\
(\%)\end{array}$ & $\begin{array}{c}\text { HS } \\
(\%)\end{array}$ \\
\hline Q16/Q65 & 1,7 & $-5,5$ & $-7,6$ & 2,3 \\
Q16/Q80 & 1,1 & $-2,5$ & $-8,1$ & 1,8 \\
Q16/Q86 & 0,9 & 0,9 & 0,2 & 11,0 \\
Q16/Q75 & $-0,2$ & $-9,5$ & $-14,5$ & $-5,3$ \\
Q16/Q06 & $-0,5$ & $-14,3$ & $-14,6$ & $-5,4$ \\
Q16/Q81 & $-0,8$ & $-9,1$ & $-9,1$ & 0,7 \\
Q16/Q87 & $-2,1$ & $-24,1$ & $-25,4$ & $-17,4$ \\
Q65/Q80 & 2,3 & 9,8 & 1,9 & 12,8 \\
Q65/Q86 & 1,2 & $-10,7$ & $-12,6$ & $-3,2$ \\
Q65/Q75 & 0,7 & $-5,5$ & $-12,0$ & $-2,6$ \\
Q65/Q06 & $-0,4$ & $-6,0$ & $-7,6$ & 2,3 \\
Q65/Q81 & -1.5 & $-43,0$ & $-43,8$ & $-37,8$ \\
Q65/Q87 & $-1,0$ & $-14,5$ & $-17,2$ & $-8,3$ \\
Q80/Q86 & 1,6 & $-6,0$ & $-11,3$ & $-1,8$ \\
Q80/Q75 & 1,1 & $-5,4$ & $-10,4$ & $-0,8$ \\
Q80/Q06 & 0,4 & $-16,2$ & $-20,4$ & $-11,8$ \\
Q80/Q81 & 0,0 & $-17,5$ & $-17,1$ & $-8,2$ \\
Q80/Q87 & 0,5 & $-11,9$ & $-12,6$ & $-3,2$ \\
Q86/Q75 & 2,0 & 10,9 & 5,0 & 16,2 \\
Q86/Q06 & 1,7 & 5,1 & 4,9 & 16,2 \\
Q86/Q81 & 0,5 & $-13,1$ & $-12,9$ & $-3,5$ \\
Q86/Q87 & $-0,5$ & $-19,3$ & $-20,5$ & $-12,0$ \\
Q75/Q06 & 1,5 & $-14,6$ & $-18,9$ & $-10,2$ \\
Q75/Q81 & 1,3 & $-12,9$ & $-12,5$ & $-3,1$ \\
Q75/Q87 & 0,3 & $-20,4$ & $-21,1$ & $-12,6$ \\
Q06/Q81 & 2,1 & 0,3 & 0,8 & 11,6 \\
Q06/Q87 & 1,6 & 5,7 & 4,4 & 15,6 \\
Q81/Q87 & 2,2 & 5,2 & 4,3 & 15,5 \\
\hline & & & &
\end{tabular}

\section{Kết Luận}

Kết quả đánh giá khả năng phối phối hợp riêng dựa trên tính trạng năng suất của 28 tổ hợp lai bằng phương pháp lai diallen của 8 dòng khổ qua thế hệ $\mathrm{I}_{6}$ (Q16, Q65, Q80, Q86, Q75,
Q06, Q81), chọn được 04 tổ hợp lai triển vọng có khả năng phối hợp riêng cao có giá trị lớn hơn 2,0 và có ưu thế lai cao vượt giống đối chứng từ $10 \%$ trở lên phục vụ công tác sản suất gồm tổ hợp lai Q65/Q80: 12,8\%, Q86/Q75: 16,2\%, Q06/Q81:11,6\% và Q81/Q87: 15,5\%.

\section{Tài Liệu Tham Khảo (References)}

AHRD (High-tech Agricultural Reasearch and Development Center). (2016). The Process of bitter gourd (Momordica charantia L.) cultivation. Ho Chi Minh City, Vietnam: AHRD.

AHRD (High-tech Agricultural Reasearch and Development Center). (2015). Growing plants in substratesin a net house. Ho Chi Minh City, Vietnam: AHRD.

Griffing, B. (1956). Concept of general and specific combining cubility in relation to diallen crossing systems. Australian Journal of Biological Sciences 9(4), 463493.

Phan, K. T. (2006). Plant breeding curriculum. Ho Chi Minh City, Vietnam: Agricultural Publishing House.

Phan, P. D. T. (2017). Research on cultivar selection of hybrid bitter gourds F1 suitable to the Southeast region of Vietnam. Ho Chi Minh City, Vietnam: Research Institute for Biotechnology and Environment.

To, T. T. T., Hoang, H. D., Le, M. T. T., Tran, L. V., Nguyen, P. T . B., Huynh, T. Q., \& Thai, B. T. (2019). Evaluation of bitter gourds (Momordica charantia L.) inbred lines for breeding ( $4^{\text {rd }}$ year). Ho Chi Minh City, Vietnam: Research and Development Center for Hitech Agriculture.

Tran, H. T. M. (2017). Evaluation combining cbility of bitter gourds (Momordica charantia L.) and identification of hybrid combinations bitter gourds prospects in Cam My, Dong Nai (Unpublished master's thesis). Nong Lam University, Ho Chi Minh City, Vietnam.

Verma, R. S., \& Singh, S. S. (2014). Combining ability and gene action in indigenous bitter gourd (Momordica charantia L.). HortFlora Research Spectrum 3(2), 122126. 